\title{
SOBRE LA FLORA Y VEGETACION LIQUÉNICAS DE LAS LAVAS BÁSICAS DEL SURESTE DE ESPAÑA
}

\author{
José María EGEA y Xavier LLIMONA
}

\begin{abstract}
RESUMEN. Sobre la flora y vegetación liquénicas de las lavas básicas del Sureste de España. Las lavas básicas que afloran en distintos puntos del sureste de España, en condiciones más o menos térmicas y áridas, presentan comunidades de líquenes que se diferencian de las que se observan sobre lavas ácidas, en la misma zona, en diversos aspectos: a. Presencia de especies nitrófilas preferentemente silicícolas. b. Alta representación de especies calcícolas raramente observadas sobre rocas silíceas. c. Ausencia de numerosas especies típicas de las rocas silíceas ácidas del SE de España. d. Dominancia de las comunidades del Peltulion euplocae y del Caloplacion irrubescentis y ausencia de las del Dimelaenion radiatae y Lecanorion montagnei. Se incluye una lista de las 88 especies reconocidas. Se describe como nueva la subasociación Acarosporetum heuflerianae subas. caloplacetosum teicholytae.
\end{abstract}

Palabras clave. Flora, vegetación, líquenes, lavas básicas, SE de España.

SUMARY. On the lichen flora and vegetation of alkaline lava from southeastern Spain. The outcrops of alkaline volcanic rock from southeastern Spain, in a more or less thermic and arid climatic conditions, show lichen communities contrasting with that studied on acid lava in the same region in: a. Presence of nitrophilous species, mainly silicicolous. b. High presence of calcicolous species, rarely seen on siliceous acid rocks. c. Lack of good number of species typical of the siliceous rocks of SE Spain. d. Prevalence of communities of Peltulion euplocae and Caloplacion irrubescentis, and absence of them included in the alliances Dimelaenion radiatae and Lecanorion montagnei. A list of 88 species recognized is also afforded. A new subassociation, Acarosporetum heuflerianae subas. caloplacetosum teicholytae is described.

Key words. Flora, vegetation, lichen, alkaline lava, SE of Spain.

\section{INTRODUCCIÓN}

En comparación con las islas del Mar Menor y Cerros del Campo de Cartagena (Llimona \& Egea 1984), los afloramientos volcánicos de los alrededores de la ciudad de Murcia están constituidos por rocas a menudo oscuras, densas y básicas (fortunitas, metabasitas, ofitas, ...). La vegetación liquénica saxícola, a menudo rica, que se instala en ellas, llama la atención por la abundancia de especies eutrófilas e, incluso, basófilas. A pesar de ello, y excepto en los puntos en los que el aporte por vía atmosférica (deposición de polvo

Este estudio se enmarca dentro de los proyectos de investigación PB 90-0615-C03-01, PB 92-0795C02-01 y PB 93-1129-C02-01, financiados por la Dirección General de Investigación Científica y Técnica. 


\begin{tabular}{llcc}
\hline Localidad & UTM & A(m) & S \\
\hline $\begin{array}{l}\text { Alicante } \\
\text { 1. Isla de Nueva Tabarca }\end{array}$ & & 10 & \\
2. Túnel de Orihuela & XH2226 & 240 & $\mathrm{M}$ \\
$\begin{array}{l}\text { Albacete } \\
\text { 3. Sierra de las Cabras }\end{array}$ & & \\
$\quad$ (Cáncarix) & XH2353 & 671 & $\mathrm{~J}$ \\
Murcia & & & \\
4. Ribazo (Cehegín) & & $\mathrm{O}$ \\
5. Chaparral (Cehegín) & XH0917 & 520 & $\mathrm{O}$ \\
6. Cabecitos Negros (Fortuna) & XH1516 & 600 & $\mathrm{~F}$ \\
7. Cabezo Negro de Zeneta & XH6622 & 170 & $\mathrm{~B}$ \\
8. Barqueros & XH7807 & 150 & $\mathrm{M}$ \\
9. Fuente Aledo & XH4403 & 380 & $\mathrm{~B}$ \\
10. Cabezo Negro de Tallante & XH4865 & 400 & $\mathrm{D}$ \\
11. Barranco de la Pistolera & XH6368 & 100 & $\mathrm{R}$ \\
$\quad$ (Cabo Tiñoso) & XH6558 & 50 & \\
12. Sierra de la Carrasquilla & XH1751 & 600 & \\
$\quad$ Aguilás) & & & $\mathrm{L}$ \\
Almería & & 30 & \\
13. Cortijo González (Vera) & XH0519 & & \\
\hline
\end{tabular}

Tabla 1. Localidades estudiadas. Abreviaturas: A: altitud, S: substrato, M: metabasitas, J: jumillitas, O: ofitas, F: fortunitas, V: veritas, B: basalto, D: diabasa, R: riodacitas, L: lamproíta. Localities studied.

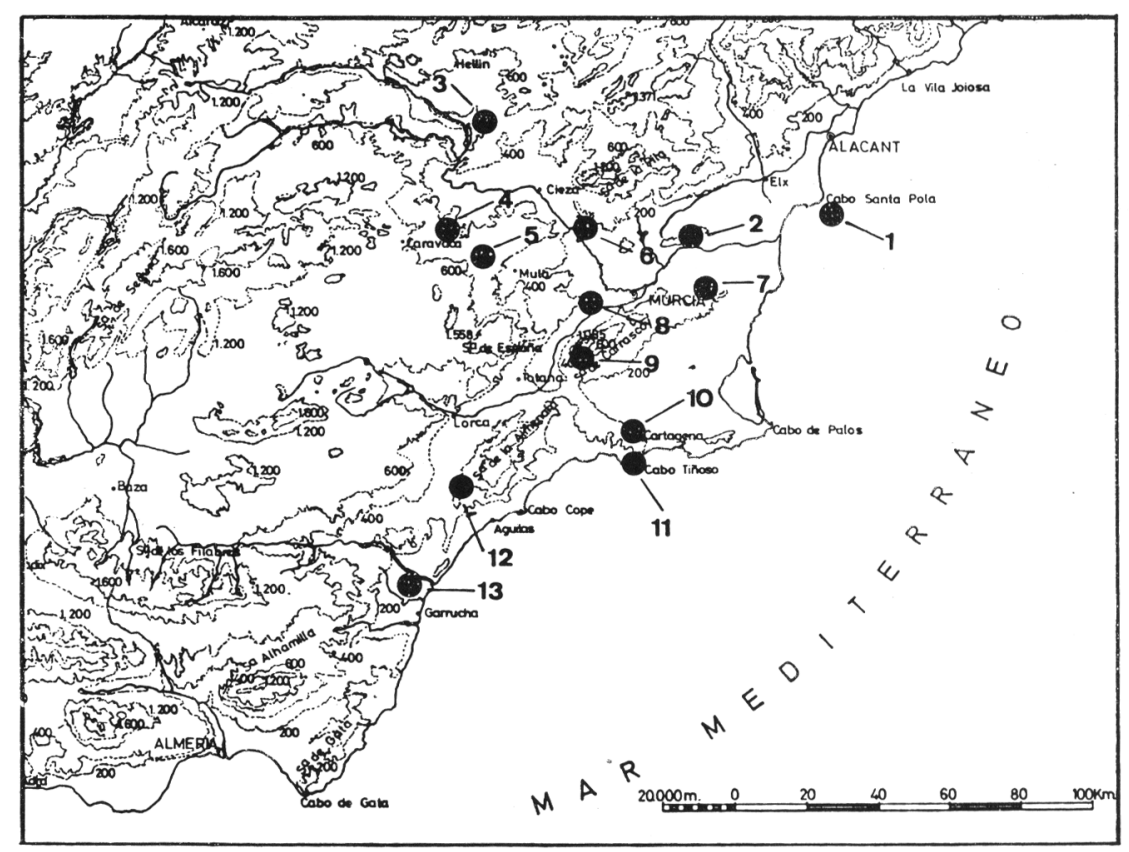

Fig, 1. Localidades estudiadas

Fig. 1. Localidades estudiadas. Localities studied. 
carbonatado) es muy elevado, el poblamineto liquénico parece bien relacionado con el Acarosporetum heuflerianae Llimona \& Egea 1987, incluido en la alianza Caloplacion irrubescentis Llimona \& Egea 1987, aunque a escasa distancia de la alianza Dimelaenion radiatae Llimona 1975. Algunos taxones poco conocidos confieren originalidad a la flora de estos ambientes. Ello, y el interés de precisar los conceptos de líquenes silicícolas, basófilos, neutrófilos y de rocas carbonatadas, justifican una prospección detenida de estos substratos.

Todas las localidades estudiadas (Tabla 1, Fig. 1) están situadas en los pisos termo y mesomediterráneo, con ombroclimas semiárido y seco.

\section{METODOLOGÍA}

Este trabajo complementa los de Egea y Llimona (1987, 1994), y sigue la misma metodología. El material testigo se halla depositado en el herbario de la Universidad de Murcia (MUB).

\section{RESULTADOS}

Las comunidades observadas pueden ser incluidas entre las que se mencionan a continuación:

Acarosporetum heuflerianae Llimona \& Egea 1987 subas. caloplacetosum teicholytae subas. nova

Tipo nomenclatural: Inv. 1, tabla 2.

Comunidad de superficies soleadas, horizontales o medianamente inclinadas, ricas en nutrientes. Se diferencia de la subasociación típica, descrita sobre rocas ácidas (Egea \& Llimona 1987) por la elevada presencia de Buellia dispersa, Lecanora muralis, Aspicilia radiosa y sobre todo, por la entrada de varias especies nitrófilas de Caloplaca, como: $C$. teicholyta, $C$. aetnensis, $C$. conversa y $C$. conglomerata (veáse tab. 2).
Buellio-Caloplacetum littoreae Egea \& Llimona 1984

Comunidad muy bien representada en la Isla Plana o de Nueva Tabarca (Alicante), única localidad donde se ha encontrado esta asociación sobre lavas básicas. Presenta la misma ecología y composición florística que en las lavas ácidas del Mar Menor, en Murcia (Llimona \& Egea 1984), de donde fue descrita (veáse tabla 3 ).

Peltuletum obscuranto-euplocae Llimona \& Egea 1985

Comunidad de superficies de escorrentía. Dentro de la alianza Peltulion euplocae Llimona \& Egea 1985, es la asociación más termófila, propia del piso termomediterráneo del sureste de España (Llimona \& Egea 1985). Se encuentra en contacto con la asociación Acarosporetum heuflerianae (veáse tabla 4).

\section{FLORA Y COROLOGÍA}

Los datos florísticos corológicos se han condensado en el anexo I. Cada localidad va encabezada por un número que coincide con el empleado en la lista de localidades estudiadas (tab. 1) y en el mapa correspondiente (fig. 1).

\section{CONCLUSIONES}

Se detecta una especial abundancia de especies nitrófilas, más o menos subcosmopolitas y relativamente frecuentes sobre las rocas ácidas (sean éstas volcánicas o no). Entre ellas, podemos destacar: Acarospora heufleriana, Aspicilia radiosa, Buellia dispersa (= Buellia tergestina), Caloplaca irrubescens, Candelariella vitellina, Lecanora muralis, Parmelia pulla y Xanthoria calcicola.

Es especialmente característico el elevado número de especies típicamente calcícolas, que sólo en muy raras ocasiones se han observado 


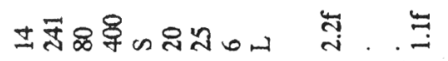

$\stackrel{m}{\tilde{N}}$.

I. +

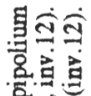

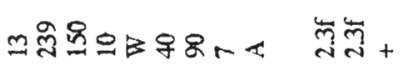

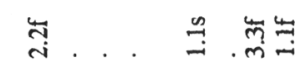

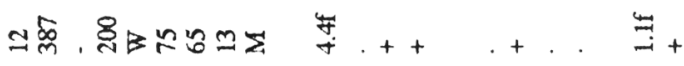

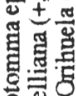

을을

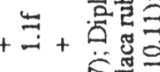

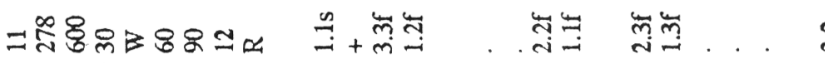

完兽

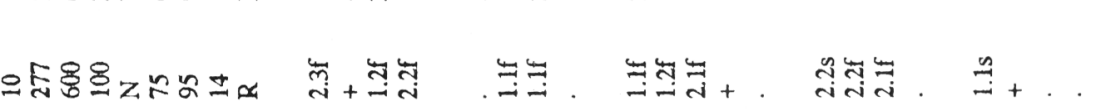

㟧

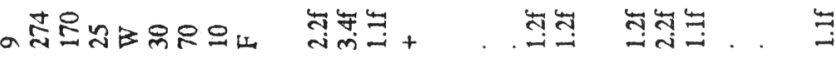

过

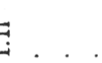

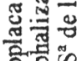

तु

तิ

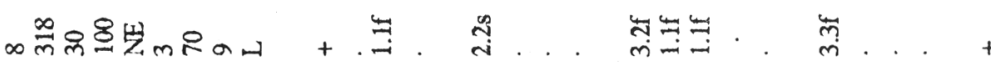

完首

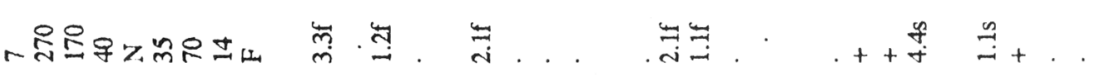

a

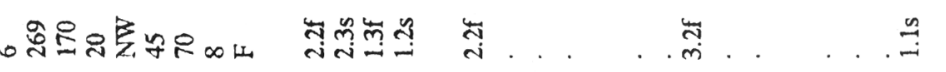

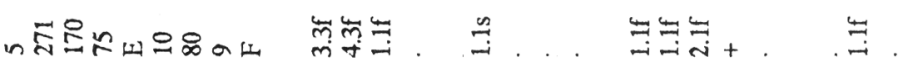

. .

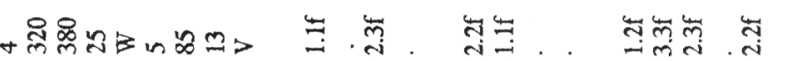

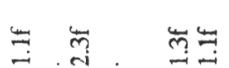

硧

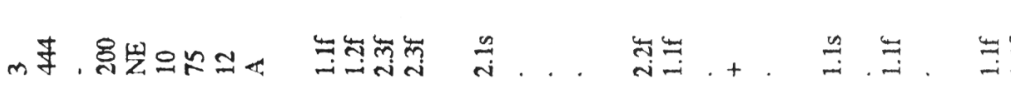

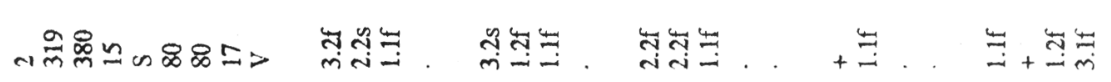

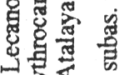

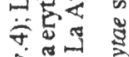

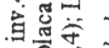

did.

过然

差.

跑

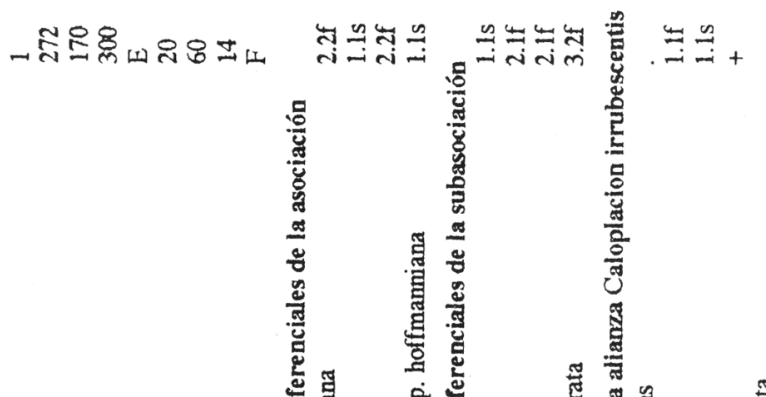
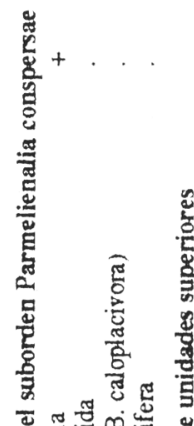

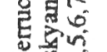

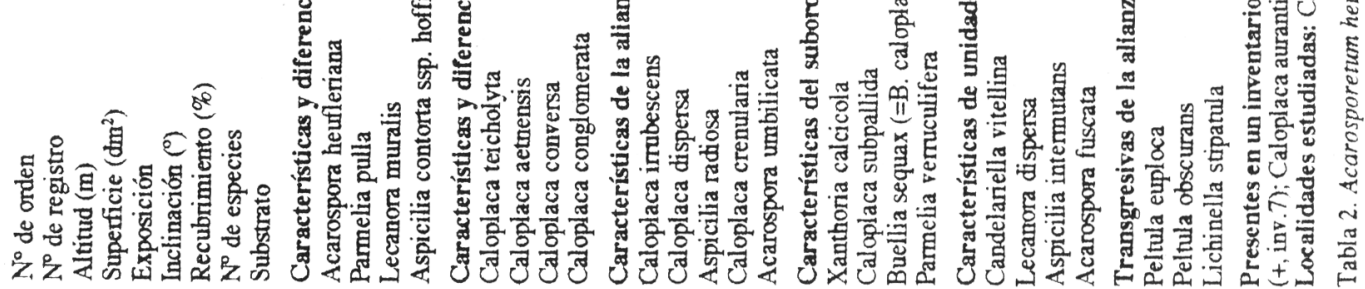




\begin{tabular}{|c|c|c|c|c|}
\hline$N^{o}$ de orden & 1 & 2 & 3 & 4 \\
\hline $\mathrm{N}^{\mathbf{o}}$ de registro & 392 & 389 & 391 & 388 \\
\hline Altitud (m) & 5 & 4 & 15 & 10 \\
\hline Supérficie $\left(\mathrm{dm}^{2}\right)$ & 50 & 100 & 40 & 5 \\
\hline Exposición & $\mathrm{S}$ & $\mathrm{N}$ & $\mathrm{N}$ & S \\
\hline Inclinación $\left({ }^{\circ}\right)$ & 5 & 50 & 3 & 5 \\
\hline Recubrimiento (\%) & 90 & 70 & 80 & 100 \\
\hline $\mathrm{N}^{\mathrm{o}}$ de especies & 7 & 4 & 4 & 5 \\
\hline Caloplaca littorea & $1.1 \mathrm{f}$ & $2.2 \mathrm{f}$ & $3.2 f$ & $3.2 f$ \\
\hline Buellia sequax & & & & \\
\hline (=B.caloplacivora) & $1.1 \mathrm{f}$ & $2.4 \mathrm{f}$ & $3.2 \mathrm{f}$ & $3.2 \mathrm{f}$ \\
\hline Lecania turicensis & $3.2 \mathrm{f}$ & & $1.1 \mathrm{f}$ & $2.2 \mathrm{f}$ \\
\hline Caloplaca flavescens & $4.3 \mathrm{f}$ & & & $1.2 \mathrm{f}$ \\
\hline Diploicia subcanescens & & $3.4 \mathrm{~s}$ & & \\
\hline Caloplaca irrubescens & $1.2 \mathrm{f}$ & $1.1 \mathrm{f}$ & & \\
\hline Lecanora lisbonensis & $1.1 \mathrm{f}$ & & & \\
\hline Candelariella vitellina & $1.1 \mathrm{f}$ & . & & \\
\hline Lecanora dispersa & & & + & $1.1 \mathrm{f}$ \\
\hline
\end{tabular}

Localidades estudiadas: Isla Plana o de Nova Tabarca (Inv. 1-4).

Tabla 3. Buellio-Caloplacetum littoreae Llimona \& Egea 1984 en las rocas ácidas de la región, como: Aspicilia cernohorskyana, Caloplaca aurantia, $C$. citrina, C. chalybaea, C. decipiens, C. erythrocarpa, $C$. saxicola, $C$. teicholyta, $C$. variabilis, Diploschistes muscorum, Diplotomma epipolium, Fulgensia fulgida, F. subbracteata, Lecania turicensis, Lecanora lisbonensis, Psora testacea, P. vallesiaca, Rinodina bischoffii, Squamarina cartilaginea y Toninia sedifolia.

Es bastante localizada, en general, la presencia de algunas especies que tienen su óptimo en zonas costeras del piso termomediterráneo y termocanario, como: Acarospora maroccana, Dimelaena radiata, Diploicia subcanescens, Llimonaea occulta, Pertusaria gallica y Rinodina beccariana.

Sorprende la ausencia de numerosas especies y comunidades de las lavas ácidas y rocas silíceas en general del sureste de España,

\begin{tabular}{|c|c|c|c|c|}
\hline $\mathrm{N}^{0}$ de orden & 1 & 2 & 3 & 4 \\
\hline $\mathrm{N}^{\circ}$ de registro & 445 & - & - & 273 \\
\hline Altitud (m) & 200 & 600 & 240 & 170 \\
\hline Superficie $\left(\mathrm{dm}^{2}\right)$ & 100 & 100 & 30 & 100 \\
\hline Exposición & W & $\mathrm{N}$ & W & S \\
\hline Inclinación $\left({ }^{\circ}\right)$ & 30 & 40 & 50 & 35 \\
\hline Recubrimiento (\%) & 60 & 40 & 50 & 75 \\
\hline $\mathrm{N}^{\circ}$ de especies & 7 & 7 & 5 & 8 \\
\hline Peltula euploca & $2.1 \mathrm{~s}$ & $2.2 \mathrm{~s}$ & $2.1 \mathrm{~s}$ & $\theta^{\circ}$ \\
\hline Lichinella stipatula & $1.2 \mathrm{~s}$ & $1.1 \mathrm{~s}$ & $1.1 \mathrm{~s}$ & $1.1 \mathrm{~s}$ \\
\hline Peltula obscurans & $2.2 \mathrm{f}$ & . & $2.2 \mathrm{f}$ & $3.2 \mathrm{f}$ \\
\hline Lichinella cribellifera (=Rechingera c.) & $3.2 \mathrm{f}$ & & $1.1 \mathrm{f}$ & $1.1 \mathrm{f}$ \\
\hline Peltula omphaliza & . & $1.1 \mathrm{f}$ & $1.1 \mathrm{f}$ & . \\
\hline Peltula placodizans & . & + & . & . \\
\hline Caloplaca irrubescens & $1.2 \mathrm{f}$ & + & . & + \\
\hline Aspicilia contorta ssp. hoffmanniana & $1.1 \mathrm{f}$ & + & . & $3.2 \mathrm{f}$ \\
\hline Xanthoria calcicola & + & . & . & . \\
\hline Acarospora heufleriana & . & + & . & $1.2 \mathrm{f}$ \\
\hline Aspicilia radiosa & . & . & . & $2.3 \mathrm{f}$ \\
\hline Caloplaca teicholyta & . & . & . & $1.1 \mathrm{~s}$ \\
\hline
\end{tabular}

Localidades estudiadas: La Atalaya (inv. 1); Sierra de la Carrasquilla (inv. 2); Sobre el túnel de Orihuela (inv. 3); Cabecitos Negros de Fortuna (inv. 4). 
entre las que cabe mencionar: a. Los géneros Dirina, Opegrapha, Ramalina y Roccella. b. Especies como: Acarospora charidema, Buellia fimbriata $(=B$. cerussata $)$, Lecanora schistina, Protoparmelia montagnei, Rhizocarpon lusitanicum, Xanthoria resendei y Caloplaca scoriophila. Faltan todas las comunidades de las alianzas Dimelaenion radiatae Llimona 1975, Lecanorion montagnei Llimona 1987 y
Roccellion phycopsis Egea \& Llimona em. Egea 1989.

La ausencia de estos taxones y sintaxones puede ser debida más a la falta de condiciones microclimáticas favorables que a la naturaleza del substrato. Por ejemplo, éste sería el caso de las especies y comunidades ombrófobas, atribuible a la ausencia de superficies protegidas de la lluvia.

\section{ANEXO I: CATÁLOGO FLORÍSTICO}

\section{Localidades}

Acarospora fuscata (Nyl.) Arnold

A. heufleriana Körber

A. maroccana B. de Lesd.

A. microcarpa (Nyl.) Wedd.

A. scotica Hue

A. umbilicata Bagl.

Aspicilia cernohorskyana (Clauz. \& Vezda) Roux

A.contorta (Hoffm.) Krempelh. ssp hoffmanniana Eckman et Froberg

A. intermutans (Nyl.) Arnold

A. radiosa (Hoffm.) Poelt \& Leuckert (=Lobothallia r.)

Buellia dispersa Massal. (=B.tergestina)

B. sequax (Nyl.) Zahlbr. (=B.caloplacivora Llimona et Egea)

B. stellulata (Tayl. in Mack.) Mudd.

Caloplaca aetnensis B. de Lesd.

C. aractina (Fr.) Häyrén

C. aurantia (Pers.) Steiner

C. chalybaea (Fr.) Miill. Arg.

C. citrina (Hoffm.) Th. Fr.

C. conglomerata (Bagl.) Jatta

C. conversa (Krempelh.) Jatta

C. crenularia (With.) Laundon (=C.festiva)

C. decipiens (Arnold) Blomb. \& Forss.

C. erythrocarpa (Pers.) Zwackh

C. flavescens (Hudson) Laundon (=C.heppiana)

C. irrubescens (Arnold) Zahlbr.

C. littorea Tavares

C. rubelliana (Ach.) Lojka

C. saxicola (Hoffmann) Nordin

C. subpallida Magnusson

C. teicholyta (Ach.) Steiner

C. variabilis (Pers.) Müll. Arq.

Candelariella vitellina (Hoffm.) Müll. Arq.

Catapyrenium contumescens (Nyl.) Breuss

Catillaria chalybeia (Borrer) Massal.

Cladonia pyxidata (L.) Hoffm.

Dimelaena radiata (Tuck.) Hale \& Culb.

Diploicia subcanescens (Werner) Hafel. \& Poelt

Diplochistes actinostomus (Pers. ex Ach.) Zahlbr.

D. candidissimus (Krempelh.) Zahlbr.

D. muscorum (Scop.) R. Sant.

D. scruposus (Schreber) Norman

Diplotomma epipolium (Ach.) Arnold

D. glaucoatrum (Nyl.)

Endocarpon pusillum Hedwig.

Fulgensia fulgida (Nyl.) Szat.

F. subbracteata (Nyl.) Poelt

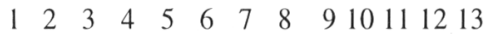

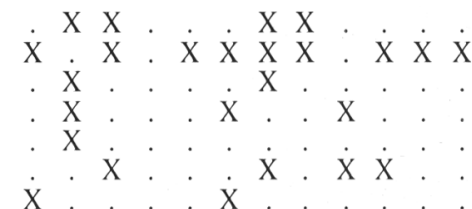

$\begin{array}{lllllllllllllllll}X & \dot{X} & \dot{X} & \dot{X} & \dot{X} & X & \dot{X} & \dot{X} & \dot{X} & \dot{X} & \dot{X}\end{array}$

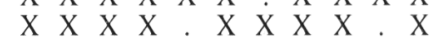

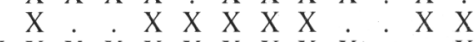

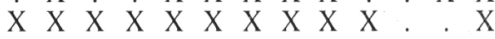

$X X \cdot X \cdot X$. $X X X \dot{X}$.

$\dot{\mathrm{X}} \dot{\mathrm{X}} \cdot \dot{\mathrm{X}} \cdot \dot{\mathrm{X}} \cdot \dot{\mathrm{X}} \cdot$

$\dot{\mathrm{X}} \dot{\mathrm{X}} \dot{\mathrm{X}} \dot{\mathrm{X}} \dot{\mathrm{X}} \dot{\mathrm{X}}$

. $\mathrm{X} \cdot \mathrm{X} \cdot \mathrm{X} \cdot \mathrm{X} \cdot \mathrm{X}$

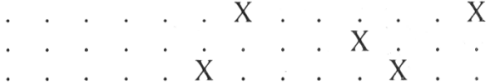

$\cdot \dot{\mathrm{X}} \cdot \mathrm{X} \cdot \dot{\mathrm{X}} \cdot \mathrm{X}_{\mathrm{X}} \cdot$

. $\dot{X} \cdot \mathrm{X} \cdot \mathrm{X} \dot{\mathrm{X}} \cdot \dot{\mathrm{X}} \mathrm{X} \mathrm{X} \dot{\mathrm{X}}$

$\cdot \dot{X} \cdot \mathrm{X} \cdot \dot{X} \cdot \dot{X} \cdot \dot{X} \cdot \dot{X} \cdot$

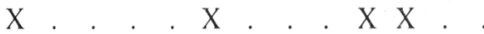

$\mathrm{X} \dot{\mathrm{X}} \cdot \dot{\mathrm{X}} \mathrm{X} \dot{\mathrm{X}} \dot{\mathrm{X}} \mathrm{X} \mathrm{X} \dot{\mathrm{X}} \dot{\mathrm{X}}$

$\mathrm{X}$

$\dot{\mathrm{X}} \cdot \dot{\mathrm{X}} \cdot \dot{\cdot} \cdot \dot{\mathrm{X}} \cdot \dot{\cdot} \cdot \cdot \cdot$

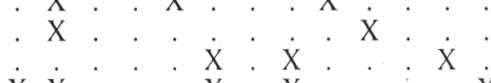

$\mathrm{X} \mathrm{X} \cdot \mathrm{X} \cdot \mathrm{X} \cdot \mathrm{X}$

$\dot{\mathrm{X}} \cdot \dot{\mathrm{X}} \mathrm{X} \cdot \dot{\mathrm{X}} \dot{\mathrm{X}} \dot{\mathrm{X}} \mathrm{X} \cdot \dot{\mathrm{X}} \dot{\mathrm{X}}$

${ }_{\dot{X}} \mathrm{X} X \cdot \mathrm{X}_{\mathrm{X}} \mathrm{X} X \cdot \mathrm{X} X$

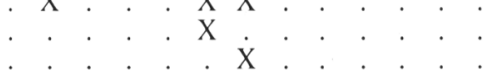

$\mathrm{X} \cdot \mathrm{X} \cdot \mathrm{X} \dot{\mathrm{X}}$.

$\dot{\mathrm{X}} \cdot \dot{\mathrm{X}} \dot{\mathrm{X}} \cdot \dot{\mathrm{X}} \cdot \dot{\mathrm{X}} \cdot$

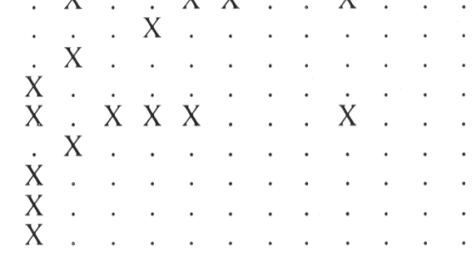




\section{Localidades}

Hafëllia leptoclinoides (Nyl.) Scheideg. \& Mayrh.

Lecania turicensis (Hepp) Müll. Arg.

Lecanora campestris (Schaer.) Hue

L. dispersa (Pers.) Sommerf.

L. gangaleoides Nyl.

L. lisbonensis G. Samp.

L. muralis (Schreb.) Rabenh.

L. rupicola ssp. subplanata (Nyl.) Leuck. \& Poelt

Lecidella asema (Nyl.) Knoph \& Hertel (=L.subincongrua)

L. carpathica Körber.

Lepraria nivalis Laundon (=L.crassissima)

L. stipatula Nyl.

Llimonaea occulta Egea \& Torrente

Ochrolechia parella (L.) Massal.

Parmelia conspersa (Ehrh. ex Ach.) Ach.

P. pulla Ach.

P. tiliacea (Hoffm.) Ach.

$\mathrm{P}$. verruculifera $\mathrm{Nyl}$.

Peltula euploca (Nyl.) Poelt ex Pisut

P. obscurans (Nyl.) Gyeln.

P. omphaliza (Nyl. in Eckf.) Wetm.

P. placodizans (Zahlbr.) Wetm.

Pertusaria gallica B. de Lesd.

P. monogona Nyl.

Physconia enteroxantha (Nyl.) Poelt

Polysporina simplex (Dav.) Vezda

Psora testacea Hoffm.

P. vallesiaca (Schaer.) Timdal

Rhizocarpon geographicum (L.) DC.

Rinodina beccariana Bagl. (=R.subglaucescens (Nyl.) Sheard)

R. bischoffii (Hepp) Massal.

R. gennarii Bagl.

R. trachytica (Massal.) Bagl. \& Car.

Rinodinella dubyanoides (Hepp) Mayrh. \& Poelt

Squamarina cartilaginea (With.) P. James

Tephromela atra (Huds.) Hafellner

Toninia sedifolia (Scop.) Timdal (=T.coeruleonigricans)

Verrucaria lecideoides (Massal.) Trevisan

V. nigrescens Pers.

Xanthoria calcicola Oxner
Lichinella cribellifera (Nyl.) Moreno \& Egea (=Rechingera c.)

Physcia dubia (Hoffm.) Lettau

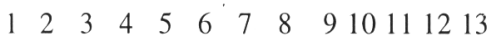

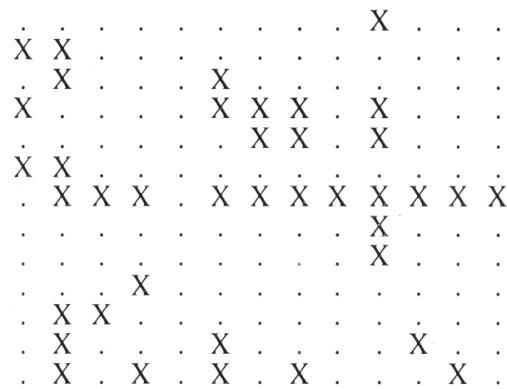

$\dot{\mathrm{X}}$

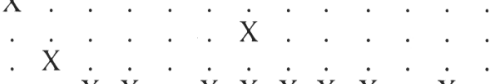

$\cdot \dot{x} \dot{X} \cdot \dot{x} \dot{x} \dot{X} \dot{x} \dot{x} \cdot \dot{X}$

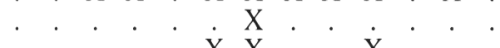

$\cdot \dot{\mathrm{X}} \dot{\mathrm{X}} \dot{\mathrm{X}} \cdot \mathrm{X} \mathrm{X} \dot{\mathrm{X}} \cdot{ }_{\mathrm{X}}^{\mathrm{X}} \dot{\mathrm{X}}$

. $\mathrm{X} \cdot \mathrm{X} \cdot \mathrm{X} \cdot \mathrm{X} \cdot \mathrm{X} \mathrm{X} \cdot \mathrm{X}$

$\mathrm{X} \cdot \mathrm{X} \cdot \mathrm{X} \cdot \mathrm{X} \cdot \mathrm{X}$

$\cdot \dot{x} \cdot \dot{\mathrm{X}} \cdot \dot{\mathrm{X}} \cdot \mathrm{X} \cdot$

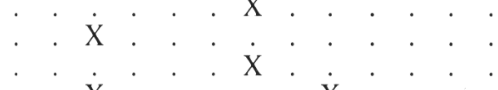

$\cdot \dot{\mathrm{X}} \cdot \cdot \cdot \mathrm{X} \cdot \dot{\mathrm{X}} \cdot \cdot \dot{\mathrm{X}}$

$\dot{\mathrm{X}} \dot{\mathrm{X}} \cdot \dot{\mathrm{X}} \cdot \mathrm{X}$

$\dot{\mathrm{X}} \cdot \dot{\mathrm{X}} \cdot \dot{\mathrm{X}} \cdot \dot{\mathrm{x}} \cdot \dot{\mathrm{x}}$

.

$\dot{\mathrm{X}}$
$\dot{\mathrm{X}} \dot{\mathrm{X}}$
$\dot{\mathrm{X}} \cdot \dot{\mathrm{X}}$

\section{BIBLIOGRAFÍA}

EGEA, J.M. y X. LLIMONA -1987- Las comunidades de líquenes de las rocas silíceas no volcánicas del SE de España. Acta Bot. Barcinonensia 36: 1-123.

EGEA, J.M. \& X. LLIMONA- 1994- La flore et la végétation lichéniques des laves acides du parc naturel de la Sierra del Cabo de Gata (SE de l'Espagne) et des régions voisines. Bull. Soc. Linn. Provence. Homm. sci. à C. Clauzade 45: 263-281.

LLIMONA, X. y J.M. EGEA -1984- La vegetación liquénica saxícola de los volcanes del Mar Menor (Murcia, S.E. de España). Butll. Inst. Cat. Hist. Nat. (sec. Bot.) 51: 77-99.
LLIMONA, X. y J.M. EGEA -1985- Las comunidades liquénicas de las superfícies de escorrentía de las rocas silíceas mediterráneas. Anales Jard. Bot. Madrid 4, 41(2): 429-444.

Aceptado para su publicación en Febrero de 1997

Dirección de los autores. J.M. EGEA: Departamento de Biología Vegetal (Botánica); Facultad de Biología, Universidad de Murcia, Campus de Espinardo, 30071 Murcia, España. X. LLIMONA: Departamento de Biología Vegetal (Botánica), Facultad de Biología, Universidad de Barcelona, Diagonal 645, Barcelona 08028, España. 